Voix et Images

voixetimages

\title{
Roland Barthes fragmentaire
}

Jean-Louis Major

Volume 16, numéro 1 (46), automne 1990

Les correspondants littéraires d'Alfred DesRochers

URI : https://id.erudit.org/iderudit/200882ar

DOI : https://doi.org/10.7202/200882ar

Aller au sommaire du numéro

\section{Éditeur(s)}

Université du Québec à Montréal

\section{ISSN}

0318-9201 (imprimé)

1705-933X (numérique)

Découvrir la revue

\section{Citer cet article}

Major, J.-L. (1990). Roland Barthes fragmentaire. Voix et Images, 16(1), 150-153. https://doi.org/10.7202/200882ar d'utilisation que vous pouvez consulter en ligne.

https://apropos.erudit.org/fr/usagers/politique-dutilisation/ 


\section{Roland Barthes fragmentaire}

\section{par Jean-Louis Major, Université d'Ottawa}

Un souvenir. Paris, octobre 1968: la rentrée à l'École pratique des hautes études. Dans la grande salle vétuste, l'auditoire attend, incertain. Les conversations s'effilochent. Barthes est là, cigarillo en coin, imperturbable, le regard entre l'intérêt et l'ennui. Malgré les séquelles de mai, chacun veut renouer. Dans les illuminations du Quartier latin, on a contesté les formes d'enseignement, au nom de la spontanéité. Ailleurs, quelques irréductibles tutoient les professeurs, par principe. Â l'École pratique, de toute façon, il n'y eut jamais officiellement que des séminaires, pas de cours, ni de professeurs - des directeurs d'études. Barthes commence par faire l'éloge du séminaire, puis dissèque le mythe de la spontanéité, y décelant la banalité, le cliché. Il enchaîne avec le relevé des codes dans Sarrazine, lexie par lexie: ce qui deviendra $\mathbf{S} / \mathbf{Z}$. Le discours du maître sans le magistral, pourrait-on ironiser en le paraphrasant. L'épisode n'en recoupe pas moins l'une des constantes de l'œuvre.

Explorant la conscience la plus aiguë des codes discursifs comme mode de signification, Barthes nourrit à leur endroit un rapport de fascination-répulsion. S'il entretint quelque temps - notamment dans le Degré zéro de l'écriture - l'illusion qu'on pouvait les neutraliser et que la modernité en particulier se donnait les moyens de se tenir hors d'eux, il se reconnaît plutôt dans une pratique qui n'emprunte les discours que pour les détourner, de l'intérieur en quelque sorte, pour s'en détacher en s'y inscrivant. Cette pratique fait constamment retour sur elle-même, en son cours aussi bien qu'au-delà - si tant est qu'on puisse lui assigner une extériorité. Décalant la position du sujet en son énonciation même, l'écriture de Barthes incorpore en outre sa propre théorisation, établit son mode de lecture et se redouble, se prolonge ou se retourne (comme on retourne un vêtement) dans la réflexion ou l'interview. Aucun de ses livres dont le titre ne se retrouve entre guillemets. Barthes annule la frontière entre l'écriture et le commentaire, mais du même coup, et du même mouvement qui la fonde, il récuse toute lecture de son œuvre. S'il jouait peut-être (pédagogiquement?) l'effarement quand on le citait à lui-même, l'attitude n'avait rien d'une feinte.

Dans Lire le fragment ${ }^{1}$, Ginette Michaud présente un Roland Barthes fragmentaire, le terme étant à prendre hors de son acception la plus courante, aux antipodes donc d'un fragment d'une totalité visée ou à venir, qui prétendrait tenir en un lieu (ou tenir lieu de) Roland Barthes, sa doctrine, sa méthode. En fait, c'est en s'opposant à cette visée totalisante que sa lecture se tient au plus près de ce que serait une "vérité" 
de Roland Barthes. Dans sa conclusion, c'est encore la tentation interprétative que débusque Lire le fragment, se demandant si, malgré tout, cette volonté de puissance ne se serait pas immiscée inconsciemment en son propos, pour se parler à travers lui. C'est marquer tout ensemble la difficulté du dessein et le mode selon lequel Ginette Michaud s'y adonne.

Le fragment, qui de prime abord se donne pour ce qui s'oppose au discours, est devenu l'emblème de la modernité: depuis les Romantiques d'Iéna, depuis Barthes surtout. D'abord thématisée, la fragmentation reçoit sa formulation théorique dans Plaisir du texte, pour être ensuite constamment reformulée en même temps que pratiquée: la question du fragment traverse toute l'œuvre de Barthes et emprunte diverses formes et divers noms avant de s'actualiser dans le fragmentforme [...]. Tous les livres de Barthes sont, plus ou moins, écrits sous forme de fragments. Mais le titre de l'ouvrage de Ginette Michaud, programmatique, annonce et appelle d'abord une lecture du fragment. C'est à une écriture que s'attache Ginette Michaud, pour interroger les conditions et les modalités de sa lecture, puis, dans un deuxième temps, la pratique de Barthes, en deux livres: Roland Barthes par Roland Barthes et Fragments d'un discours amoureux.

Ginette Michaud pose d'emblée que le fragment n'est pas un genre: ni partie (d'un tout), ni forme, ni maxime, ni fétiche, ni détail. Chacune de ces négations en forme de paradoxe s'inscrit du même coup dans le refus d'une approche. Mais la négation n'est pas exclusion: un déplacement plutôt.

Le fragment n'est pas, n'est plus un objet. Il n'occupe plus un champ réservé à la philosophie, à la littérature, à la psychanalyse. [mais il] trouve son articulation à la jonction de la philosophie, de la littérature et de la psychanalyse, entre ces divers champs du savoir.

D'où cette précision importante: Ginette Michaud n'étudie pas le fragment en tant que forme, mais bien plutôt l'écriture dú fragment, ce qu'elle nomme, selon un tour heureux comme il s'en retrouve tant en cet ouvrage, le fragment(aire), c'est-à-dire le fragment en tant que pratique, en tant que questionnement, en tant qu'exigence. Fragmentable plutôt que fragmenté, voilà, pour nous, le fragment.

Se tenant hors de sa définition en quelque sorte, comme des angles qui l'abordent et des champs auxquels il appartient, le fragment appelle un nouveau mode de lecture.

Conçue comme un travail de liaison et d'organisation qui prolonge les qualités de l'œuvre [...] en s'y identifiant mimétiquement, la lecture est alors censée vouloir la dévoiler, l'éclairer, tout en effaçant elle-même ses propres opérations critiques. 
Comment alors lire le discontinu sans l'annuler? Par entrées, par effractions, en multipliant les ruptures critiques, en les soulignant, mais en évitant le mimétisme que serait une écriture par fragments. La lecture du fragmentaire ne peut s'effectuer que par la mise en question et le déplacement topologiques.

Du Roland Barthes, Ginette Michaud prélève des séries de fragments: les photos de l'album, la théorisation interne, les anamnèses, mais aussi la série des brouillons et la page couverture comme fragment. Chaque fois, elle en interroge l'effet - et l'affect - dans une lecture qui "incompose" le livre, tout en s'accomplissant ellemême par tranches successives. Sa lecture se resserre ensuite d'un cran théorique, pour se saisir en tant que transfert, selon le modèle même (textuel et analytique) des Fragments d'un discours amoureux. Multipliant ses propres entrées, elle s'attache à celles du texte: débuts, départs, titres, cadres introductifs et inducteurs, dédicaces, scènes premières (primitives) règlent les rapports entre le livre et le fragment, le sujet de l'écriture et le lecteur. Son propos, délibératif (s'il y a ceci, il y a cela), progresse par déplacements perspectifs. Son approche adopte volontiers la forme du retardement (avant de... il faut...) et du retour (pour remettre en question, pour lever de nouvelles pistes, pour relancer les possibles, plutôt que pour résumer).

Dès le titre du Roland Barthes, auquel Ginette Michaud s'arrête dans une tranche intitulée "Interruption: le nom fracturé", le fragmentaire est à l'œuvre. Le livre figure dans la collection "Écrivains de toujours", dont les titres adoptent une forme commune: $X$ (l'auteur étudié, présenté) par lui-même. Y figurent, par exemple, un Malraux par lui-même de Gaëtan Picon, un Montaigne par lui-même de Francis Jeanson. Dans le cas de l'ouvrage consacré à Barthes, à l'opposé de la fiction d'auto-écriture qu'est le "par lui-même" des autres ouvrages de la collection, c'est vraiment l'auteur qui s'étudie, se présente. Pourtant, le titre change, même si ailleurs Barthes désigne le livre sous la forme habituelle Barthes par lui-même. Sur la couverture, on lit: roland BARTHES par roland barthes. Le jeu des caractères, des minuscules, des capitales travaille le redoublement du nom propre. Le nom de l'auteur sujet du livre (objet d'une présentation, d'une étude) se redouble du nom de l'auteur du livre, c'est-à-dire de l'auteur en position de sujet. Mais la place normalement occupée par le nom de l'auteur du livre demeure vide. Selon un mouvement topologique tant de fois pratiqué, sous diverses formes et à divers titres, chez Barthes, l'auteur en tant que sujet s'est déplacé. En évacuant la position de sujet pour se redoubler dans le titre, le nom de l'auteur se fragmente comme référent, alors que le titre comme totalisation du livre, comme maîtrise d'une totalité par un sujet, s'est retourné sur lui-même (contre lui-même ?). Comment lire ce titre ? Comment même l'écrire, puisque l'étendue de l'italique en est incertaine? Et surtout, comment lire ce 
livre de fragments qu'il désigne? La réception en a fait une lecture autobiographique: lieu de vérité, foyer interprétatif de l'œuvre. Ainsi, Philippe Lejeune y opère un classement thématique selon lequel l'espace autobiographique se partage en types de récits. Mais ce n'est pas lire Barthes ni le fragmentaire.

Je feins d'écrire sur ce que j'ai autrefois écrit, écrit Barthes dans le Roland Barthes. Et dans ce que Ginette Michaud nomme la scène d'in(tro)duction des Fragments d'un discours amoureux, pour en mettre au jour tous les enjeux: c'est donc un amoureux qui parle et qui dit. En chacun de ces livres, Barthes ne pose les termes d'un contrat de lecture que pour le déjouer et pour en établir un autre, plus exigeant. Il y revalorise l'imaginaire, au sens lacanien du terme, en s'y inscrivant comme sujet, mais pour pratiquer une écriture qui annule le discours, autobiographique dans le cas du Roland Barthes, amoureux dans le cas des Fragments... Plus précisément, il n'effectue la mise en scène de ces discours que pour y opérer le déplacement du sujet en son énonciation même.

L'écriture fragmentaire suscite à son tour un déplacement analogue du lecteur - au masculin, au neutre plus précisément, puisqu'il s'agit du champ de la lecture plutôt que d'une personne, alors que le sujet de l'énonciation dans Lire le fragment est le "nous" féminin singulier, tout ensemble abstrait, individualisant et marque du discours institutionnel. En réalité, Ginette Michaud fait jouer tous ces plans à la fois: elle cite ses. modèles - Blanchot, Derrida, Felman, Barthes surtout et l'abondante littérature qu'il a suscitée - toujours avec à propos, sa démarche s'en inspire, mais elle est d'abord à l'écoute de sa propre lecture; elle déploie un savoir théorique irréprochable sur lequel son interrogation prend appui, mais elle suit le parcours de sa propre écriture. Les enjeux d'une lecture du fragmentaire sont inséparables, chez elle, du jeu de l'intelligence critique.

On ne résume pas l'ouvrage de Ginette Michaud, ni totalisation ni livre (mimétique) par fragments: c'est la marque de sa réussite tout autant que de sa nature. Véritable lecture du grain de l'écriture, Lire le fragment est une pratique qui s'interroge dans et par son accomplissement même. Il n'est pas un acquis sur quoi établir une connaissance (de Barthes, du fragment, de la rhétorique du fragment), mais un acte de conscience incontournable, une question qui ne s'abolit pas de se poser ni de se donner des réponses, théorie inachevée de l'inachèvement. Par l'éclectisme de l'intelligence critique, par la conscience en acte des ressources et des exigences du langage dans l'exercice de sa propre écriture, Ginette Michaud prolonge et renouvelle la démarche de Barthes autant qu'elle l'interroge.

1. Ginette Michaud, Lire le fragment. Transfert et théorie de la lecture chez Roland Barthes, Montréal, Hurtubise HMH, 1989, 321 p.(Brèches). 